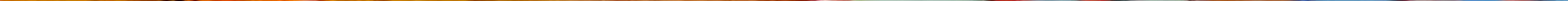




\title{
8 El reto del envejecimiento de la población. Algunas reflexiones
}

\author{
Raúl Hernández Castellón*
}

\begin{abstract}
RESUMEN. Desde el punto de vista demográfico el mundo ha transitado por importantes cambios durante el siglo XX. La reducción de los niveles de mortalidad y de fecundidad. En el caso de la primera ésta determinó uno de los hechos más importantes de la segunda mitad del siglo XX: el envejecimiento de la población. La evolución de las citadas variables demo-gráficas varió según el nivel de desarrollo económico y social de los países. En el caso cubano, la mortalidad y la fecundidad se redujeron a un ritmo mayor que en el resto de los países subdesarrollados, determinando así una mayor incidencia en el envejecimiento de su población. Los cambios determinados por el triunfo de la Revolución en 1959, aceleraron la reducción de los niveles de ambas variables, provocando también un rápido envejecimiento de su población, a tal punto que en el año 2025 estará muy cerca de los países con mayor nivel de envejecimiento del mundo, y para el 2050, estará entre los primeros. El aumento de las personas de 60 años y más a un ritmo muy rápido, impone la necesidad de atender dicho reto, en particular el de la atención de esas personas desde todos los puntos de vista. En el presente trabajo se abordan estas y otras importantes cuestiones.
\end{abstract}

Palabras clave: Envejecimiento, fecundidad, mortalidad, adulto mayor, demografía, hogar de anciano, crecimiento de la población, atención social, atención de salud.

SUMMARY. From the demographic viewpoint the world has gone through significant changes during the twentieth century; the reduction of mortality and fertility levels. Regarding the first, it determined one of the most important events of the second half of the twentieth century: the aging of the population. The evolution of the aforementioned demo-graphic variables varied depending on the level of economic and social development of the countries. In the Cuban case, mortality and fertility declined at a faster rate than the rest of the underdeveloped countries, thus determining a higher incidence in the aging of their population. The changes determined by the triumph of the 1959 Revolution, accelerated the reduction in the levels of both variables, also causing rapid aging of its population, to the extent that in 2025 will be very close to the countries with the highest level of aging in the world, and by 2050, will be among the first. The increase in 60 year old people and over a very fast rate imposes the need to address this challenge, particularly the care of these people from all points of view. This paper addresses these and other important issues.

Keywords: Aging, fertility, mortality, elderly, demography, nursing home, population growth, social care, health care.

\section{Introducción}

Las cuestiones relacionadas con el envejecimiento de la población y las personas de edad han tenido un papel destacado en las tres principales conferencias internacionales sobre población organizadas por las Naciones Unidas durante el último cuarto del siglo pasado. Por ejemplo, en la última de ellas, celebrada en el Cairo, Egipto, en 1994, se reconoció que la incidencia social y económica del envejecimiento de la población constituía tanto una oportunidad como un reto para las sociedades ${ }^{1}$.
En la Segunda Asamblea Mundial de Envejecimiento, celebrada en Madrid², en 2002, se indicaron algunos aspectos sobre esta temática, entre ellos que:

- Es evidente que el mundo se halla en proceso de transformación debido a unos cambios demográficos sin precedentes, los cuales tienen sus orígenes en los siglos XIX y XX y que continúan todavía en el actual siglo XXI. Los descensos de la fecundidad, reforzados por una creciente longevidad, han producido y continúan produciendo, cambios sin precedentes en las estructuras de todas las sociedades, en especial la histórica inversión

Recibido enero 2011/Aceptado y versión final marzo de 2011.

* Dr. en Ciencias Geográficas, Universidad de La Habana, Cuba.raul@cedem.uh.cu

1. Naciones Unidas 1994: Población y Desarrollo, vol. 1: Programa de Acción adoptado en la Conferencia Internacional sobre la Población y el Desarrollo, El Cairo, 5 al 13 de septiembre de 1994. Núm. De venta: S.95.XII.7).

2. Naciones Unidas (2002): Informe de la Segunda Asamblea Mundial sobre el Envejecimiento. A/CONF.197/9. Nueva York. 
que se ha producido en la proporción de jóvenes y personas de edad. Las consecuencias, profundas generales y duraderas del envejecimiento de la población, brindan enormes oportunidades a todas las sociedades, pero también imponen enormes retos.

- Como el ritmo de envejecimiento de la población es mucho más rápido en los países en desarrollo que en los países desarrollados, los países en desarrollo tendrán menos tiempo para adaptarse a las consecuencias del envejecimiento de la población. Además, este proceso en los países en desarrollo se produce en niveles de desarrollo socioeconómico muy inferiores a los que existían en su momento en los países desarrollados.

- La mayoría de las personas de edad son mujeres, ya que ellas superan a los hombres en términos de la esperanza de vida. En el 2000, había 63 millones más de mujeres que hombres de 60 años ó más, y en las edades más avanzadas, la supremacía es de dos a cinco veces.

El objetivo del presente trabajo es el de llevar a cabo un breve análisis, básicamente desde el punto de vista demográfico, del gran reto que representa el proceso de envejecimiento en el mundo, su incidencia por regiones, en particular en América Latina y Cuba.

\section{Metodología}

Se describe la evolución de las variables demográficas, en particular la fecundidad y la mortalidad, que son las principales variables asociadas con el envejecimiento de la población. Se acomete el análisis del tratamiento del referido envejecimiento en las conferencias internacionales de Viena, Austria, en 1982 y de Madrid, en 2002.

Se pasa revista al envejecimiento de la población en el mundo y sus regiones. Posteriormente se realiza un breve análisis de su proceso en Latinoamerica. El referido análisis incluye la importancia de su incidencia, tanto en el pasado reciente, como en el futuro: el último cuarto del siglo pasado, y primer cuarto del presente siglo, e incluso a mediados del mismo. Asimismo, se caracterizan los países según grandes grupos de su grado de envejecimiento.
A continuación se presenta el análisis de las características del envejecimiento de la población en Cuba, incluyendo su evolución por provincias. Asimismo, se enfoca la atención social (incluida la atención de salud) al adulto mayor, el surgimiento y desarrollo de la Universidad del Adulto Mayor, en muchas provincias del país. La referida atención social también comprende el análisis de los beneficiarios de la seguridad social y de la asistencia social.

\section{Contexto del estudio}

\subsection{El Plan de Acción Internacional sobre Envejeci- miento de la Población}

En la citada Segunda Asamblea Mundial de Envejecimiento se adoptó el Segundo Plan de Acción Internacional sobre envejecimiento de la Población ${ }^{3}$.

En la introducción al Plan de Acción aprobado, se indican varias cuestiones importantes:

- Que en el pasado siglo XX se produjo una revolución de la longevidad. También se plantea que la notable transición demográfica que se está produciendo hará que para mediados de siglo los porcentajes de la población mundial correspondientes a viejos y jóvenes sean iguales.

- Que el envejecimiento de la población pasará a ser una cuestión de primordial importancia en los países en desarrollo que, según se proyecta, envejecerán rápidamente en la primera mitad del siglo XXI.

- Que es indispensable integrar el proceso del envejecimiento mundial, que está en curso de evolución, en el proceso más amplio del desarrollo.

- Que este plan requiere cambios de las actitudes, las políticas y las prácticas a todos los niveles y en todos los sectores, para que puedan concretarse las enormes posibilidades que brinda el envejecimiento en el siglo XXI.

- Que las recomendaciones para la adopción de medidas se organizan con arreglo a tres orientaciones prioritarias: las personas de edad y el desarrollo; fomentar la salud y el bienestar hasta llegada la vejez; y crear entornos propicios y favorables.

3. IBIDEM. 
Cabría preguntarse: ¿Cuáles son las principales características demográficas de este proceso? A continuación se abordan estas cuestiones.

\subsection{El envejecimiento y el contexto internacional}

En el mundo, la proporción de personas de 60 años y más no registró cambios muy importantes entre 1900 y 1950, a pesar de la gran reducción de la fecundidad en los países desarrollados. Sin embargo, durante el período 1950-2000 tuvo lugar una elevación de dicha proporción de forma un poco más pronunciada, asociada con el comienzo de la reducción de la fecundidad en los países subdesarrollados. Estos últimos representaban en 1950 el 68\% de la población mundial, y en el año 2000 ya constituían las cuatro quintas partes (ver gráfico No.1).

La reducción de la fecundidad se puede visualizar, a través de la evolución de las tasas de crecimiento de los menores de 15 años, así como su otro extremo, el de la población de 60 años y más. En las últimas décadas, el rápido proceso de envejecimiento de la población mundial, ha determinado el estudio de la denominada población de la "cuarta edad", es decir, 80 años y más.

En los países en los cuales la fecundidad ha experimentado reducciones bastante importantes, las tasas de crecimiento de la población 15 años y más han descendido considerablemente o ya muestran valores negativos, en tanto sucede totalmente lo contrario con las de los adultos mayores (ver gráfico No.2).

Gráfico No. 1. Tres siglos de envejecimiento mundial de la población

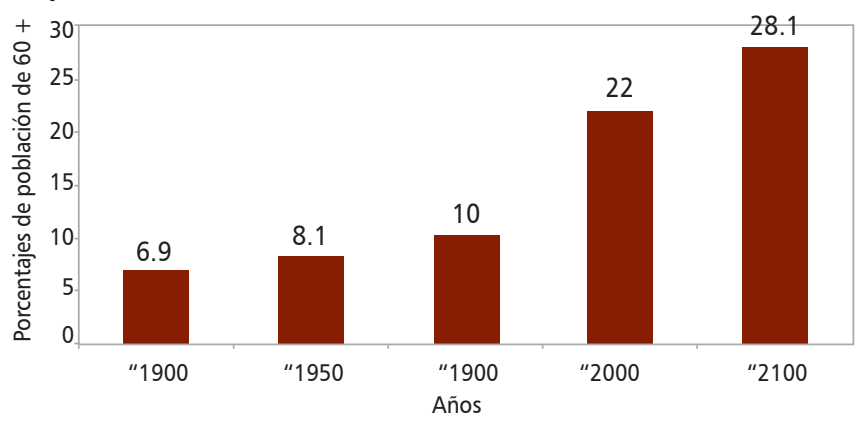

Fuente: United Nations (1999): World Population Prospects, the 1998 Revision. New York.
Según se indicó en las primeras páginas de este documento, una de las principales características del envejecimiento de la población en el mundo y en la mayoría de los países, es la preponderancia de las mujeres entre las personas de edad avanzada, lo cual es una cuestión que tenderá a acentuarse con el tiempo, debido a la prevalencia de la sobremortalidad masculina (ver gráfico 3).

Tal como se mencionó, debido a la sobremortalidad masculina, aumentará, por tanto, el número de viudas, que implicará a su vez más soledad para ellas. Por otra parte, crecerá mucho más el rol de cuidadoras de las adultas mayores, lo cual incluye no sólo a sus esposos muy mayores, sino también a otros parientes, además de sus nietos y bisnietos.

La relación entre la esperanza de vida media y el diferencial hombres-mujeres, basada en estimados para 1999 se muestra en el gráfico No. 4. Mientras que la tendencia hacia el incremento de los diferenciales por sexo en la mortalidad con la disminución general de la mortalidad es aparente en términos generales, en el gráfico No.4 se muestran desviaciones muy marcadas. En particular, sobre todo alrededor de un nivel de esperanza de vida de 65 años, no existe una clara relación, con la

Gráfico No. 2. Tasas medias anuales de crecimiento de la población total por regiones del mundo y grandes grupos de edades. 2000-2050

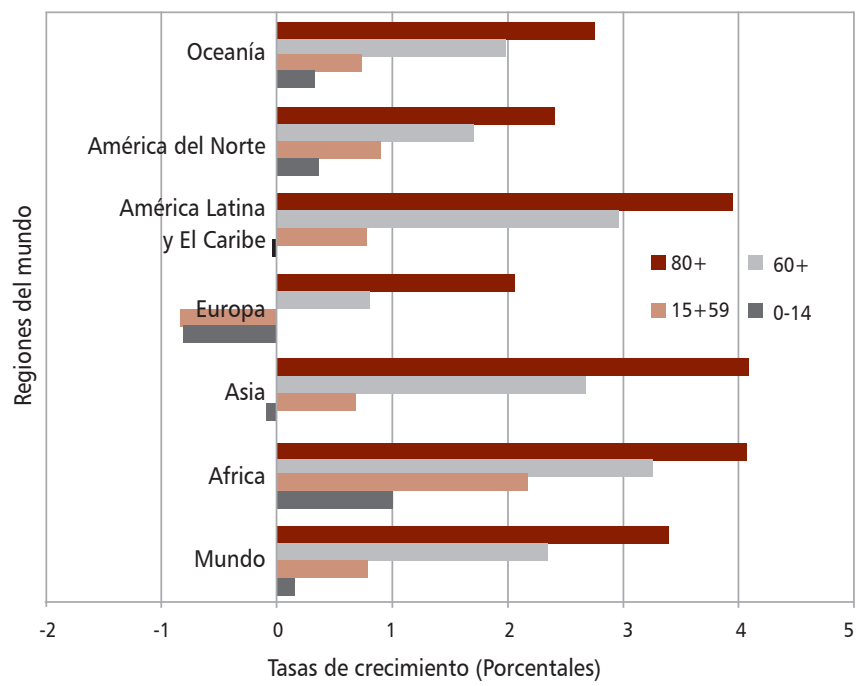

Fuente: Rodríguez, V. Hernández, R. Puga, D. Características demográficas y socioeconómicas del envejecimiento de la población en España y Cuba. Consejo Superior de Investigaciones Científicas. Monografías 26. Madrid, 2003. Pág. 13. 
Gráfico No. 3. Proporción de mujeres entre grupos de edades seleccionados en todo el mundo. 2000

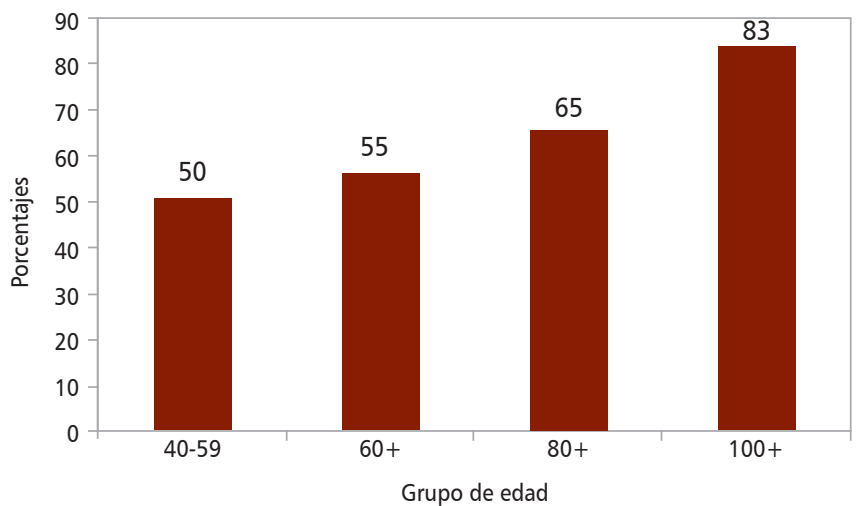

Fuente: Naciones Unidas 2001: Envejecimiento de la población mundial: 1950-2050. Resumen Ejecutivo. Departamento de Asuntos Económicos y Sociales. División de Población. ST/ESA/SER.A/20207/ES. Nueva York.

ventaja femenina en la esperanza de vida, que se mueve en un rango de virtualmente cero hasta más de 12 años a niveles promedio de esperanza de vida de alrededor de 70 años. Todos los países con diferenciales de sexo extremos (10 ó más años) pertenecían a la antigua Unión Soviética.

3.3. El envejecimiento en América Latina y el Caribe: rasgos principales

Las tasas de crecimiento mostradas en el Gráfico No.2, confirman el grado de aceleración que ha adquirido el proceso de envejecimiento en los países subdesarrolla-
Gráfico No. 4. Años de diferencia entre la esperanza de vida masculina y femenina, versus esperanza de vida por país, 1999

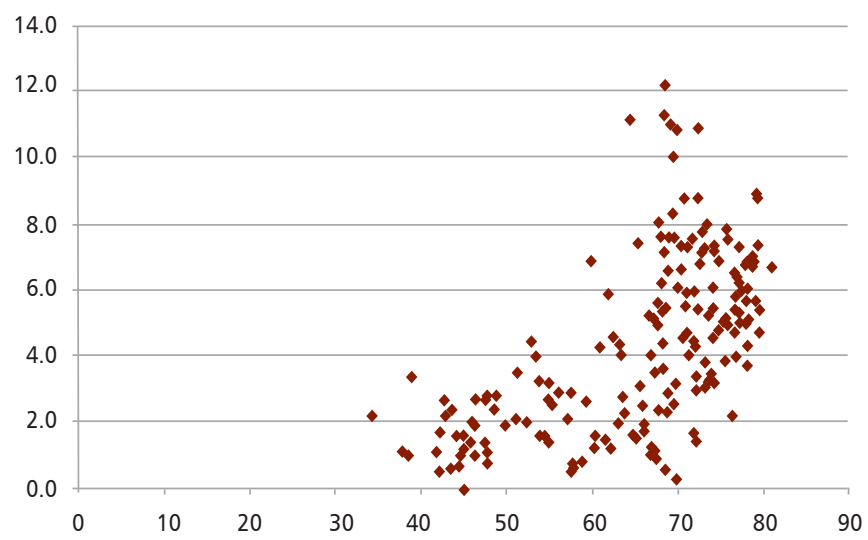

Fuente: 1999: A.D. Lopez, J.Salomon, O. Ahmad, C. J.L. Murray, D. Mafat. Life Tables for 191 countries: Data, Methods and Results. GPE Discussion Paper Series: No. 9. EIP/GPE/ EBD. GPE Discussion Paper Series: No. 9. World Health Organization.

dos, el cual se está llevando a cabo a un ritmo mayor que el que tuvo lugar en los países desarrollados.

En comparación con el resto de las regiones subdesarrolladas del mundo, América Latina y el Caribe se encuentran entre las que muestran un mayor envejecimiento de su población, en general. Esta afirmación se verifica si se observa el futuro crecimiento de la población de 60 años y más durante el período 1950-2050, que condicionó las perspectivas del siglo XXI, incluyendo el análisis de las sub regiones latinoamericanas (Cuadro No.1).

Cuadro No.1. América Latina y el Caribe: población y tasas de crecimiento de 60-74 y 75 años y $+1950-2050$. Miles de personas y porcentajes

\begin{tabular}{|c|c|c|c|c|c|c|c|c|}
\hline & \multicolumn{8}{|c|}{ Población: (Miles de personas) } \\
\hline & \multicolumn{2}{|c|}{ América Latina } & \multicolumn{2}{|c|}{ Caribe } & \multicolumn{2}{|c|}{ América Central } & \multicolumn{2}{|c|}{ América del Sur } \\
\hline & $60-74$ & $75 y+$ & $60-74$ & $75 y+$ & $60-74$ & $75 y+$ & $60-74$ & $75 y+$ \\
\hline 1950 & 8213 & 1706 & 945 & 224 & 1974 & 420 & 5294 & 1063 \\
\hline 1975 & 16577 & 4381 & 1745 & 447 & 3195 & 1056 & 11637 & 2879 \\
\hline 2000 & 31399 & 9835 & 2720 & 1029 & 6816 & 2185 & 21865 & 6640 \\
\hline 2025 & 73214 & 24191 & 5392 & 2092 & 17032 & 5786 & 50791 & 16311 \\
\hline \multirow[t]{2}{*}{2050} & 120764 & 60427 & 7474 & 4398 & 32162 & 16295 & 81125 & 39735 \\
\hline & \multicolumn{8}{|c|}{ Tasas de crecimiento (Porcentajes) } \\
\hline $1950-75$ & 2,67 & 3,52 & 2,38 & 2,66 & 1,88 & 3,45 & 3,00 & 3,69 \\
\hline $1975-00$ & 2,47 & 3,07 & 1,75 & 3,15 & 2,89 & 2,79 & 2,44 & 3,16 \\
\hline $2000-25$ & 3,20 & 3,38 & 2,62 & 2,72 & 3,43 & 3,61 & 3,18 & 3,37 \\
\hline $2025-50$ & 1,96 & 3,43 & 1,29 & 2,84 & 2,46 & 3,81 & 1,84 & 3,34 \\
\hline
\end{tabular}

Fuente: Rodríguez, V. Hernández, R. Puga, D. Características demográficas y socioeconómi-cas del envejecimiento de la población en España y Cuba. Consejo Superior de Investigacio-nes Científicas. Monografías 26. Madrid, 2003. Pág. 13 
Lo que más se destaca de la observación de las regiones es que América del Sur y el Caribe, respectivamente, constituían en el año 2000 las áreas de mayor y menor concentración de personas de los grupos de edades 60-74 y 75 años y más. En efecto, en ambos grupos de edades la primera área contaba en dicho año con algo más de dos tercios del total de personas de edad avanzada. Por otra parte, el Caribe sólo alcanzaba entre el 8 y el 10\% de dichas personas en el referido año.

Las tasas de crecimiento reflejan una tendencia decreciente en el siglo pasado para las personas de 60 a 74 años de edad. No obstante, como resultado de la explosión demográfica de los años 50 y 60, las tasas aumentan nuevamente durante el período 2000-2025. Una vez pasada esta etapa "pico", las tasas decrecen nuevamente durante el período 2025-2050 (ver cuadro No.2).

Otra es la situación del grupo de 75 años y más, la cual refleja la tendencia actual del proceso de envejecimiento de la población en muchas partes del mundo: el explosivo crecimiento de los así denominados "viejos más viejos", o también calificados como integrantes de la "cuarta edad", en que aumenta la disminución de la etapa activa de la vida. En general, las tasas de crecimiento de este grupo son más altas que las del grupo anterior. En muchos períodos las referidas tasas superan bastante la barrera del 3\%, es decir, valores muy altos.

La evolución mencionada implica a su vez una serie de retos para toda la sociedad, ya que disminuirá bastante el peso relativo de la población de la denominada "tercera edad", que supone un retiro activo, lo que llama a tomar desde ya las medidas necesarias para atender las crecientes necesidades de estas personas en muchas áreas como la atención de salud, las políticas de asistencia y seguridad social, entre otras. Un reciente estudio del CELADE (Centro Latinoamericano de Demografía) sobre envejecimiento de la población en América Latina utiliza una tipología para caracterizar dicha región y así también discutir el referido proceso de envejecimiento.
En la región se advierte una marcada heterogeneidad. Para captarla se puede clasificar a los países, según la etapa en que se encuentran en su proceso de envejecimiento, en tres categorías:

- Envejecimiento incipiente: países con porcentajes de personas de 60 años y más que oscilan entre el 5\% y el $7 \%$ en el año 2000 y que alcanzarían valores de entre $15 \%$ y $18 \%$ en 2050 . Incluye a países como Bolivia, Guatemala, Haití, Honduras, Nicaragua y Paraguay. Este proceso podría acelerarse si en estos países se consolida e incrementa la baja de la fecundidad.

- Envejecimiento moderado: países con porcentajes de $6 \%$ a $8 \%$ de personas de 60 años y más, que en torno del año 2050 superarán el 20\%. En este grupo se sitúan Belice, Colombia, Costa Rica, Ecuador, El Salvador, Guyana, México, Perú, República Dominicana y la República Bolivariana de Venezuela.

- Envejecimiento moderado avanzado: países que presentan en la actualidad porcentajes de personas mayores entre $8 \%$ y $10 \%$, y que experimentarán un aumento

Cuadro No. 2. Países seleccionados de América Latina y el Caribe: proporción y tasas de crecimiento de la población de 60 años y más. 2000-2050

\begin{tabular}{|l|c|c|c|c|c|}
\multirow{2}{*}{ País } & \multicolumn{3}{|c|}{$\begin{array}{c}\text { Porcentaje de población } \\
\text { de } 60 \mathrm{y}+\end{array}$} & \multicolumn{2}{c|}{$\begin{array}{c}\text { Tasa de creci- } \\
\text { miento }\end{array}$} \\
\cline { 2 - 6 } & $\mathbf{2 0 0 0}$ & $\mathbf{2 0 2 5}$ & $\mathbf{2 0 5 0}$ & $\begin{array}{c}\mathbf{2 0 0 0}- \\
\mathbf{2 0 2 5}\end{array}$ & $\begin{array}{c}\mathbf{2 0 2 5}- \\
\mathbf{2 0 5 0}\end{array}$ \\
\hline Uruguay & 17.2 & 19.6 & 24.5 & 1.2 & 1.3 \\
\hline Argentina & 13.3 & 16.6 & 23.4 & 1.9 & 1.9 \\
\hline Cuba & 13.7 & 25.0 & 33.3 & 2.6 & 0.9 \\
\hline Barbados & 13.4 & 25.2 & 35.4 & 2.8 & 1.0 \\
\hline Chile & 10.2 & 18.2 & 23.5 & 3.3 & 1.5 \\
\hline Brasil & 7.9 & 15.4 & 24.1 & 3.7 & 2.3 \\
\hline México & 6.9 & 13.5 & 24.4 & 3.8 & 2.8 \\
\hline Rep. Dominicana & 6.5 & 13.3 & 22.6 & 3.9 & 2.8 \\
\hline Bolivia & 6.2 & 8.9 & 16.4 & 3.3 & 3.4 \\
\hline Paraguay & 5.3 & 9.4 & 16.0 & 4.4 & 3.3 \\
\hline Honduras & 5.2 & 8.6 & 17.4 & 4.0 & 3.9 \\
\hline Total América Latina & 8.0 & 14.1 & 22.6 & 3.5 & 2.5 \\
\hline
\end{tabular}

Fuente: Guzmán, J.M. 2002: Envejecimiento y desarrollo en América Latina y el Caribe. CELADE. Serie Población y desarrollo Nº 28. Santiago de Chile. 
rápido de este porcentaje hasta alcanzar cifras entre $25 \%$ y $30 \%$ de personas mayores en 2050. Entre estos países están Bahamas, Brasil, Chile, Jamaica, Suriname y Trinidad y Tabago.

- Envejecimiento avanzado: países pioneros en el envejecimiento en América Latina, como Argentina, Cuba, Uruguay y varios países del Caribe: Antillas Neerlandesas, Barbados, Guadalupe, Martinica y Puerto Rico, con porcentajes actuales de personas mayores superiores al $10 \%{ }^{4}$, que en torno del año 2050 tendrán porcentajes que oscilarán entre 25 y $35 \%$.

En el último cuarto del siglo pasado, comenzó la reducción de la fecundidad en muchos países de la región, como parte integrante del proceso de transición demográfica, durante el cual la mortalidad fue la primera en iniciar dicha reducción.
La primera mitad del presente siglo será testigo de la ampliación del proceso antes mencionado, con una mayor reducción de la fecundidad y una algo menor de la mortalidad.

Lamentablemente, los progresos alcanzados desde la primera Asamblea Mundial del Envejecimiento, celebrada en Viena, Austria en 1982, no han sido de gran importancia, según se puede apreciar en el cuadro No. 3.

\section{Algunas características del envejecimiento de la población en Cuba}

El envejecimiento de la población cubana, al igual que el de algunos países suramericanos, está asociado con el comportamiento de las tres variables demográficas: fecundidad, mortalidad y migraciones. El gran proceso

\section{Cuadro No. 3. Progresos desde la primera asamblea mundial del envejecimiento}

Los resultados de una encuesta hecha a los países miembros, organizaciones intergubernamentales, entidades del sistema de Naciones Unidas y ONG (respondido por 61 estados miembros, 9 de ellos de América Latina y el Caribe y 22 organizaciones), muestran los siguientes progresos:

Desarrollo de planes y programas y mecanismos de coordinación.

Mejoramiento de los servicios de salud y vivienda.

Mayor seguridad de ingresos.

Aumento de la participación de las personas mayores.

Respecto a los agentes de cambio

La mitad de los países concuerda en que el Estado es el principal responsable en cuestiones del envejecimiento; otros dan esa misión a la familia y a los individuos. Dos tercios de los países afirman que las personas mayores participan en actividades de arte, música, literatura, cuidado de otras personas, servicios de voluntariado, etc. En el $50 \%$ de los países, personas mayores asesoran los planes de desarrollo gubernamental y comunitario; además, trabajan como capacitadores y educadores.

Dificultades y obstáculos:

La mitad de los países señala que las principales dificultades están en: a) vivienda y calidad de vida, seguridad de los ingresos y del empleo y protección del consumidor de edad, b) $57 \%$ de los países afirma que la falta de fondos es el motivo para no aplicar las recomendaciones del Plan de Viena, c) Algunos países no tienen un organismo definido a cargo de los asuntos de la vejez, d) las dependencias encargadas de los asuntos de los adultos mayores por lo regular no están dispuestas a asumir la responsabilidad de su cuidado, e) falta de coordinación entre los organismos gubernamentales, falta de experiencia en la formulación de políticas y desconocimiento del Plan de Viena, f) los organismos de Naciones Unidas afirman que los gobiernos no se dedican lo suficiente al tema del envejecimiento y señalan que la participación de las personas en políticas, programas y proyectos es insuficiente.

\section{Prioridades:}

Para la mayoría de los países el efecto de la migración sobre las personas mayores es una preocupación generalizada y otros temas son el entorno de las personas mayores: maltrato, violencia, etnias, salud, etc. Las ONG plantean la necesidad de cambiar el Plan de Acción, ampliando su esfera para reflejar los cambios demográficos, la tecnología, la investigación biomédica; además, piden articularlo con los compromisos mundiales sobre desarrollo social, mujer e incluir las preocupaciones de los países en desarrollo. La mayoría de los países reconoce a la cooperación internacional como dimensión necesaria para el logro de los objetivos del Plan de Acción y está por la cooperación multilateral. En cuanto a las prioridades de la cooperación, las dos esferas principales son la formulación, vigilancia y evaluación de las políticas y programas y la investigación de apoyo a la elaboración de políticas y programas. Para las ONG la principal prioridad es establecer una cooperación internacional en la ejecución, vigilancia, y evaluación de los programas sobre el envejecimiento; plantean como prioridad la capacitación de profesionales en salud y servicios sociales, investigaciones de apoyo a políticas y programas.

Fuente: Tomado de: Viveros Madariaga, A. Envejecimiento y vejez en América Latina y el Caribe: políticas públicas y las acciones de la sociedad. CELADE, 2001. Stgo. de Chile. 
inmigratorio que experimentó el país durante las tres primeras décadas del siglo XX, influyó bastante en el comportamiento de la estructura por edades ${ }^{5}$, por cuanto dichos migrantes tenían edades laborales, había elevada proporción de hombres entre ellos y arribó alrededor de un millón de personas, e independientemente de que muchos fueron repatriados, dicho monto fue importante.

La mortalidad es la variable que comenzó primero su descenso antes de los años cincuenta, aunque de forma moderada. Dicha declinación aumentó su ritmo entre los períodos 1950-1955 y 1995-2000, en que la esperanza de vida al nacer creció en 16.4 años.

La variable que influye más sobre el envejecimiento de la población, es decir, la fecundidad, también registró notable disminución al pasar de 1.7 a 0.7 hijas por mujer. Cabe aclarar también que desde 1978 la fecundidad cubana se encuentra por debajo del nivel de reemplazo (ver cuadro No.4).

Debido a las importantes reducciones registradas durante el período señalado, las de la primera mitad del siglo actual serán más modestas. En efecto, entre los años 2000 y 2050 la esperanza de vida al nacer sólo ganará 4.5 años.

La combinación de la evolución de las dos variables antes mencionadas, ha determinado un envejecimiento de la población a un ritmo verdaderamente acelerado.

Así, las personas de 60 años y más pasaron de algo más de 400 mil en 1950 a un millón y medio en el año 2000, con todas las implicaciones económicas y sociales que esto trae aparejado. Ya en el año 2025 serán alrededor de tres millones y en el 2050 llegarán a ser unos 3 millones seiscientos mil.
Cuadro No. 4. Cuba: dinámica de la esperanza de vida y de la tasa neta de reproducción. 1950-2050. Períodos seleccionados

\begin{tabular}{|c|c|c|}
\hline Períodos & $\begin{array}{c}\text { Esperanza de vida } \\
\text { al nacer }\end{array}$ & $\begin{array}{c}\text { Tasa neta de repro- } \\
\text { ducción }\end{array}$ \\
\hline $1950-1955$ & 59.3 & 1.7 \\
\hline $1970-1975$ & 70.7 & 1.6 \\
\hline $1995-2000$ & 75.7 & 0.7 \\
\hline $2000-2025$ & 78.4 & 0.9 \\
\hline $2025-2050$ & 80.2 & 0.9 \\
\hline
\end{tabular}

Fuente: Rodríguez, V. Hernández, R. Puga, D. Características demográficas y socioeconómicas del envejecimiento de la población en España y Cuba. Consejo Superior de Investigaciones Científicas. Monografías 26. Madrid, 2003. Pág. 24

Cuba no está ajena al proceso que se viene desarrollando desde hace algunos años en los países más envejecidos del mundo, que coinciden con ser los más desarrollados, es decir, el gran incremento de las personas de la "cuarta edad", o sea, las personas de 75 años y más ${ }^{6}$. Su crecimiento es tal, que de montos irrisorios en 1950, con el paso de los años llegarán casi a superar el de las personas de 60 a 74 años. El análisis de la dinámica de la proporción de menores de 15 años, en comparación con la proporción de personas de 60 años y más, que muestra el Cuadro No.5, proporciona un interesante

Cuadro No. 5 Cuba: informaciones diversas de la población de 60 a 74 y de 75 años y más 1950-2050, años seleccionados

\begin{tabular}{|c|c|c|c|c|c|c|c|c|}
\hline & \multicolumn{2}{|c|}{ Población } & \multicolumn{2}{|c|}{ Incremento } & \multicolumn{2}{|c|}{ I n cremento } & \multicolumn{2}{|c|}{ Tasa media anual } \\
\hline & \multicolumn{2}{|c|}{ (en miles) } & \multicolumn{2}{|c|}{ A b soluto } & \multicolumn{2}{|c|}{ Relativo } & \multicolumn{2}{|c|}{ de crecim. (\%) } \\
\hline & $60-74$ & $75 y+$ & $60-74$ & $75 y+$ & $60-74$ & $75 y+$ & $60-74$ & $75 y+$ \\
\hline 1950 & 341 & 86 & - & - - & - - & - - & -- & - - \\
\hline 1975 & 732 & 187 & 391 & 101 & 114,7 & 117,4 & 2,92 & 2,96 \\
\hline 2000 & 1085 & 444 & 353 & 257 & 48,2 & 137,4 & 1,55 & 3,26 \\
\hline 2025 & 2035 & 909 & 950 & 465 & 87,6 & 104,7 & 2,44 & 2,75 \\
\hline 2050 & 1905 & 1753 & -130 & 844 & $-6,4$ & 92,8 & $-0,26$ & 2,54 \\
\hline
\end{tabular}

Fuente: Rodríguez, V. Hernández, R. Puga, D. Características demográficas y socioeconómicas del envejecimiento de la población en España y Cuba. Consejo Superior de Investigaciones Científicas. Monografías 26. Madrid, 2003. Pág. 25.

5. Pueden consultarse entre otros trabajos: Raúl Hernández (1988): La revolución demográfica en Cuba. Instituto Cubano del Libro. Editorial de Ciencias Sociales. La Habana. CEDEM (1976): La población de Cuba. Instituto Cubano del Libro. Editorial de Ciencias Sociales. La Habana. Cuba. (2009): Población y Desarrollo. Centro de Estudios Demográficos de la Universidad de La Habana.

6. Peter Laslett (1996), propone cuatro etapas del ciclo de vida: la primera edad, vinculada con la infancia y la juventud; la segunda edad, asociada con la vida activa y reproductiva; la tercera edad relacionada con la etapa activa de retiro; finalmente, la cuarta edad que se refiere a la fase de declinación, mayor dependencia y deterioro más acelerado. Chackiel señala al respecto que "Considerado de esta manera, el término "tercera edad" no sería sinónimo de "vejez", sino que se refiere a una etapa específica de esa condición". Véase: Chackiel, Juan: "El envejecimiento de la población latinoamericana: ihacia una relación de dependencia?. CELADE - División de Población. Santiago de Chile, agosto de 2000. Pág. 10. 
ángulo del proceso de envejecimiento de la población cubana.

Así, mientras en 1950 la primera proporción era 4.9 veces superior a la segunda, en el año 2000, sólo la superaba 1.6 veces. El fuerte impacto de la reducción de la fecundidad y su incidencia en el envejecimiento de la población determinarán que el monto de jóvenes sea superado por las personas de edad avanzada en el año 2025. Cabe agregar que a finales de esta primera década del presente siglo, ya en varias provincias ha ocurrido el hecho mencionado. Dicho en términos del índice de envejecimiento: mientras en 1950 existían 20 adultos mayores por cada 100 jóvenes, en el año 2000 el valor alcanzado fue de 64 y en el 2025 habrá 156 viejos por cada joven, para llegar a 214 en el 2050 (ver cuadro No.6).

Desde otro punto de vista, es decir, el cociente de dependencia potencial, también denominado índice de dependencia potencial, que refleja el número de personas en edad de trabajar por cada persona de 60 años o más, muestra una tendencia decreciente, lo cual tendrá una incidencia importante en el régimen de seguridad social. Con el queda reflejada la carga de dependencia que afrontan los potenciales trabajadores. En el Gráfico No.5 se aprecia la incidencia del envejecimiento demográfico, que ha disminuido y seguirá disminuyendo. En el referido gráfico se muestra a la vez la dinámica de este indicador,
Gráfico No. 5. Índice de apoyo potencial 1950-2050

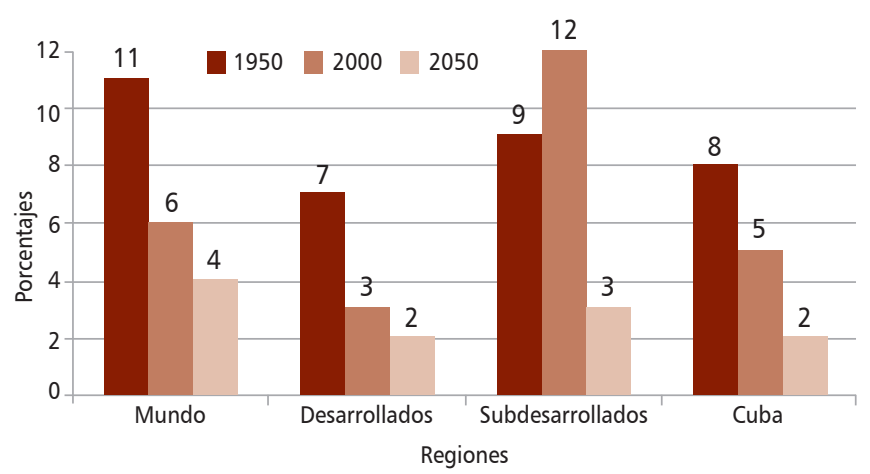

Fuente: United Nations: 1998. World Population Prospects. The 1996 Revision. Department of Economic and Social Affaires. Population Division. ST/ESA/SA/SER.A/167

comparativamente, entre Cuba, el mundo y les regiones desarrolladas y subdesarrolladas. Este país presenta una tendencia que se acerca bastante a la evolución seguida por las regiones más desarrolladas del planeta.

El aumento de la fecundidad que experimentó el país en la década de los años sesenta, con su incidencia en el aumento de la proporción de menores de 15 años, determinó el aumento del Índice de Dependencia Total (IDT) entre 1950 y 1975. En el último cuarto del siglo pasado, la fuerte reducción de la fecundidad determinó una gran reducción del referido índice. Sin embargo, el nuevo aumento del mismo (2000-2025 y 2025-2050) estuvo causado por el crecimiento del número de ancianos, los cuales superaron el monto de menores de años.

Cabe destacar que mientras que en el año 1950 sólo había una persona de 75 años por cada 5

Cuadro No. 6. Cuba: porcentajes de población por grandes grupos de edades e índices de envejecimiento y de dependencia demográfica 1970-2050

\begin{tabular}{|c|c|c|c|c|c|c|c|c|c|c|}
\hline Años & -15 & $\begin{array}{l}15- \\
59\end{array}$ & $\begin{array}{l}60- \\
74\end{array}$ & $\begin{array}{l}75 y \\
+\end{array}$ & $\begin{array}{l}\text { Edad } \\
\text { Mediana }\end{array}$ & \multicolumn{5}{|c|}{$\begin{array}{l}\text { Índices de envejecimiento y de dependencia } \\
\text { demográfica y potencial }\end{array}$} \\
\hline 1950 & 35.8 & 56.9 & 5.8 & 1.5 & 23.3 & 20.4 & 12.8 & 75.8 & 7.8 & 5.9 \\
\hline 1975 & 37.4 & 52.8 & 7.9 & 2.0 & 22.7 & 26.5 & 18.7 & 89.5 & 5.3 & 7.8 \\
\hline 2000 & 21.2 & 65.1 & 9.7 & 4.0 & 32.9 & 64.4 & 21.0 & 53.6 & 4.8 & 4.9 \\
\hline 2025 & 16.0 & 59.0 & 17.3 & 7.7 & 43.3 & 156.0 & 42.4 & 69.5 & 2.4 & 6.3 \\
\hline 2050 & 15.5 & 51.2 & 17.7 & 16.3 & 46.1 & 214.1 & 65.0 & 95.4 & 1.5 & 49.2 \\
\hline
\end{tabular}

Notas: IE $=$ Índice de envejecimiento $=60 \mathrm{y}+/<15$. IDE $=$ Índice de dependencia de los ancianos $=60 \mathrm{y}+/ 15-59$. IDT $=$ Índice de dependencia total $==<15+60 y+/ 15-59$. IAP $=$ Índice de apoyo potencial 15-59/60+ IAP1 $=$ Índice de apoyo a los padres $=80+/ 50-64$

Fuente: Rodríguez, V. Hernández, R. Puga, D. Características demográficas y socio-económicas del envejecimiento de la población en España y Cuba. Consejo Superior de Investigaciones Científicas. Monografías 26. Madrid, 2003. Pág. 27. de 60 a 74, ya en el año 2000 esa proporción pasó a casi una de cada tres, en tanto que en el año 2050 la proporción será de casi mitad y mitad.

\subsection{El envejecimiento de la po-} blación a nivel provincial

Después de analizar el proceso de envejecimiento de la población cubana a nivel nacional, procede 
ahora conocer qué ocurre a nivel provincial. El cuadro No. 7 permite ilustrar dicha situación, así como su dinámica entre 1997 y 2007.

Durante el período 1997-2007 el cambio más relevante es la disminución del peso relativo de adultos mayores residentes en Ciudad de La Habana, con relación al total del país. Lo anterior podría estar indicando cierta redistribución de los mismos, al aumentar en otras provincias. Aunque quizás con menor importancia sí sobresale el hecho de la pequeña disminución en Villa Clara, por ser ella la que muestra la mayor proporción de personas de 60 años y más del país.

Las provincias con mayor nivel de envejecimiento son Villa Clara, Ciudad de La Habana y Sancti Spíritus, las cuales incrementaron bastante sus proporciones de personas de 60 años en el período objeto de análisis. No menos substancial fue el aumento que registró Santiago de Cuba. El cuadro No.7 también responde a la pregunta de iqué provincias registran la mayor o menor congregación de adultos mayores?, o en otras palabras:
¿Dónde se ubican ellos? Ciudad de la Habana, donde se concentraba en 1997 el 19.5\% de la población total del país, a su vez albergaba el $23.6 \%$ de los adultos mayores. Sin embargo, tal como se indicó, dicho valor pasó a 21.9\% en el 2007.

Por otra parte, el nivel de urbanización de las provincias impone diferencias en las proporciones de adultos mayores según áreas de residencia. Una de ellas es el alto grado de concentración de éstos en las zonas urbanas, pues en muchas de ellas se supera el $70 \%$ y en una cifra mucho menor, traspasan la frontera del $80 \%$. Las informaciones anteriores deben ser tenidas en cuenta no sólo por los formuladores de políticas, sino también por las autoridades competentes, por los retos que implican.

De la observación del cuadro No.7 y su comparación con el cuadro No.8, se puede apreciar cuan importantes son los contrastes con las proporciones alcanzadas por los adultos mayores cubanos, dado que en el año 2007 ya 5 provincias cubanas registraron valores de $20 \%$ y más en algunos de sus municipios (18 de ellos), en tanto que 94

Cuadro No. 7. Cuba: informaciones sobre la población de 60 años y más por provincias. 1997 y 2007

\begin{tabular}{|c|c|c|c|c|c|c|c|c|c|c|}
\hline & \multirow{2}{*}{\multicolumn{2}{|c|}{$\begin{array}{c}\text { Distribución } \\
\text { Porcentual }\end{array}$}} & \multicolumn{6}{|c|}{ Proporción de personas de $60 \mathrm{y}+$} & \multirow{2}{*}{\multicolumn{2}{|c|}{$\begin{array}{c}\text { Vive en zonas } \\
\text { urbanas } \%\end{array}$}} \\
\hline & & & \multicolumn{2}{|c|}{ Ambas Zonas } & \multicolumn{2}{|c|}{ Urbana } & \multicolumn{2}{|c|}{ Rural } & & \\
\hline & 1997 & 2007 & 1997 & 2007 & 1997 & 2007 & 1997 & 2007 & 1997 & 2007 \\
\hline Cuba & 100,0 & 100,0 & 13,1 & 16,6 & 13.6 & 16.2 & 11.9 & 14.5 & 77,6 & 77,5 \\
\hline Pinar del Río & 5,9 & 6,2 & 11,8 & 15,9 & 11.6 & 15.0 & 12.2 & 14.9 & 62,5 & 63,1 \\
\hline La Habana & 6,7 & 6,6 & 14,2 & 16,5 & 14.8 & 16.5 & 12.1 & 14.6 & 81,5 & 75,9 \\
\hline Ciudad de La Habana & 23,6 & 21,9 & 15,6 & 18,9 & 15.6 & 18.9 & -- & -- & 100,0 & 100,0 \\
\hline Matanzas & 6,1 & 6,2 & 13,7 & 17,0 & 14.0 & 16.5 & 12.4 & 15.1 & 81,6 & 83,9 \\
\hline Villa Clara & 9,0 & 8,7 & 15,8 & 20,0 & 15.8 & 18.9 & 15.9 & 19.5 & 76,8 & 75,3 \\
\hline Cienfuegos & 3,7 & 3,6 & 13,6 & 16,7 & 13.6 & 15.8 & 13.7 & 16.3 & 80,5 & 80,6 \\
\hline Sancti Spìritus & 4,6 & 4,5 & 14,8 & 18,2 & 15.0 & 17.6 & 14.3 & 16.8 & 70,5 & 70,9 \\
\hline Ciego de Ávila & 3,6 & 3,5 & 13,1 & 15,7 & 13.5 & 15.5 & 12.1 & 13.6 & 76,5 & 74,2 \\
\hline Camagüey & 6,8 & 6,8 & 12,8 & 16,1 & 13.6 & 15.8 & 10.5 & 13.6 & 79,6 & 78,8 \\
\hline Las Tunas & 3,9 & 4,2 & 11,0 & 14,8 & 10.8 & 13.5 & 11.2 & 14.4 & 58,0 & 60,6 \\
\hline Holguín & 8,4 & 8,7 & 12,0 & 15,6 & 11.2 & 14.2 & 13.1 & 15.5 & 55,0 & 61,8 \\
\hline Granma & 6,1 & 6,4 & 10,8 & 14,7 & 11.2 & 14.5 & 10.2 & 12.6 & 59,7 & 62,2 \\
\hline Santiago de Cuba & 7.7 & 8,2 & 11,0 & 14,7 & 11.4 & 14.2 & 10.3 & 13.2 & 72,0 & 71,2 \\
\hline Guantánamo & 3.5 & 3,7 & 10,1 & 13,4 & 10.4 & 12.9 & 9.6 & 12.2 & 61,4 & 62,2 \\
\hline Isla de la Juventud & 0,4 & 0,6 & 7,2 & 12,0 & 7.3 & 11.1 & 6.7 & 9.1 & 88,1 & 86,6 \\
\hline
\end{tabular}

Fuente: Elaborado a partir de: ONE (1998): El envejecimiento de la población cubana. Cifras para su estudio. 1997. La Habana. ONE (2008): El envejecimiento de la población cubana. Cifras para su estudio. 2007. La Habana. 
Cuadro No. 8. Cuba: cantidad de municipios con diferentes rangos de porcentajes de población de 60 y +.1997 y 2007

\begin{tabular}{|c|c|c|c|c|c|c|c|c|c|}
\hline & \multicolumn{4}{|c|}{1997} & \multicolumn{4}{|c|}{2007} & \multirow[b]{2}{*}{ Totales } \\
\hline & $<$ de 10 & $10-14$ & $15-19$ & $20+$ & $<$ de 10 & $10-14$ & $15-19$ & $20+$ & \\
\hline Pinar del Río & & 14 & & & & 4 & 10 & & 14 \\
\hline La Habana & & 14 & 5 & & & 3 & 15 & 1 & 19 \\
\hline Ciudad de La Habana & & 7 & 8 & & & & 10 & 5 & 15 \\
\hline Matanzas & 1 & 11 & 2 & & & 2 & 11 & 1 & 14 \\
\hline Villa Clara & & 4 & 9 & & & & 5 & 8 & 13 \\
\hline Cienfuegos & & 6 & 2 & & & & 8 & & 8 \\
\hline Sancti Spiritus & & 4 & 4 & & & 2 & 5 & 1 & 8 \\
\hline Ciego de Avila & 1 & 8 & 1 & & & 5 & 5 & & 10 \\
\hline Camagüey & 2 & 11 & & & & 5 & 8 & & 13 \\
\hline Las Tunas & & 8 & & & & 6 & 2 & & 8 \\
\hline Holguín & 1 & 12 & 1 & & 1 & 3 & 10 & & 14 \\
\hline Granma & 4 & 9 & & & & 10 & 3 & & 13 \\
\hline Santiago de Cuba & 4 & 5 & & & & 6 & 3 & & 9 \\
\hline Guantánamo & 7 & 3 & & & & 10 & & & 10 \\
\hline Isla de la Juventud & 1 & & & & & 1 & & & 1 \\
\hline Totales & 21 & 116 & 32 & & 1 & 56 & 94 & 18 & 169 \\
\hline
\end{tabular}

Fuente: Elaborado a partir de: ONE (2008): El envejecimiento de la población cubana. Cifras para su estudio. 2007. La Habana. ONE (1998): El envejecimiento de la población cubana. Cifras para su estudio. 1997. La Habana.

municipios de 13 provincias reflejaron porcentajes con un rango entre 15 y 19. Esto da una clara imagen del grado de envejecimiento de la población cubana en el año 2007. La comparación con el año 1997 contribuye a confirmar aún más este hecho.

\subsection{Atención social al adulto mayor}

Se ha afirmado que el envejecimiento de la población pone en peligro el bienestar de los propios ancianos y del resto de la sociedad. El motivo sería que el diseño de la misma y de los mecanismos de solidaridad intergeneracional actuales se gestaron en momentos en que la estructura de la población era más joven y que no va a poder resistir los efectos del envejecimiento.

Los principales focos de interés de esta previsión alarmista son los gastos en pensiones y jubilaciones y los gastos en salud, ya que aumenta la proporción de quienes tienen una salud precaria. Y la preocupación se acentúa por el hecho de que aquellos que alcanzan la madurez tienen una esperanza de vida cada vez más alta, por lo que no sólo se incrementa la proporción de las personas dependientes sino que también la de los que lo van a ser durante mucho más tiempo.

La experiencia de algunos países muestra que el proceso de envejecimiento demográfico no tiene por qué ir acompañado necesariamente de una degradación de las condiciones sociales y económicas del país. En los últimos cuarenta años España ha pasado de ser un país con graves problemas estructurales al contar con un índice de desarrollo humano similar a los de Alemania y Francia, al mismo tiempo que se situaba entre los países más envejecidos del mundo. Como se ve, el auténtico factor determinante no es la evolución de la estructura por edades, sino el aumento de la riqueza y la productividad de los trabajadores españoles se ha multiplicado mucho más que el conjunto de la población o la proporción de personas que no trabajan.

Sin embargo, en América Latina, durante las dos últimas décadas del siglo pasado, se llevaron a cabo procesos de reformas del sistema de pensiones de los sectores de salud y seguridad social debidas, según se plantea, a que los antiguos sistemas mostraban deficiencias administrativas y financieras. Por otra parte, también se indica que el subsidio fiscal a dicho sistema variaba entre el 35 y el $65 \%$ del porcentaje total del gasto?

7. Guzmán, J.M. (2002): "Envejecimiento y desarrollo en América Latina y el Caribe”. Centro Latinoamericano y Caribeño de Demografía (CELADE) División de Población. Santiago de Chile, mayo de 2002. 
Concretamente, en la actualidad en varios países de América Latina los sistemas de salud (Argentina, Brasil, Chile, Costa Rica, Jamaica, México, Uruguay y Venezuela), así como los de pensiones (Argentina, Bolivia, Chile, Colombia, El Salvador, México, Perú y Uruguay) se han privatizado, por lo cual la situación de los adultos mayores atravesará diversas dificultades, según se ha planteado en algunos trabajos ${ }^{8}$. Para los trabajadores cubanos de edad avanzada, esto les trae un amargo recuerdo, pues antes de 1959 los sistemas de pensiones eran privados, en su inmensa mayoría frecuentemente amanecían sin fondos, es decir, desfalcados, y sus usuarios lanzados a la miseria. Algo similar ocurrió en México recientemente, aunque fue el caso de ahorristas, los cuales también fueron estafados y después de muchas protestas y luchas, sólo pudieron recuperar un 20\% de lo que habían ahorrado.

"La cobertura de los sistemas de jubilaciones y pensiones en Latinoamérica es muy baja, y con diferencias significativas según área de residencia. Además, los ingresos mensuales generados en la mayoría de los países son insuficientes para satisfacer las necesidades básicas de una pareja. Menos de la mitad de la población urbana de 60 años y más es beneficiaria de la seguridad social, frente a un $38 \%$ en las zonas rurales; este último valor baja apenas a $10 \%$ si excluimos a Brasil, cuya cobertura en las áreas rurales es especialmente elevada. Sólo en pocos países (Argentina, Brasil, Cuba, Chile y Uruguay) la cobertura sirve a más del 50\% de los adultos mayores. En muchos otros (Bolivia, Colombia, Ecuador, El Salvador, Honduras, México, Nicaragua, Paraguay, República Dominicana y Venezuela) no alcanza al 25\% de la población de 60 años y más"

Las pensiones en Cuba alcanzan a todos los que laboran, sin ningún tipo de distinción por sexo, raza o tipo de trabajo. Los que lo hacen en labores agrícolas reciben los mismos beneficios. Esta es una situación completamente distinta a la que atraviesan los del resto de América Latina, lo cual se reconoce en el documento citado anteriormente ${ }^{10}$. Otra ventaja de los adultos mayores cubanos es que la atención de salud es gratuita y los precios del ingreso a los hogares de ancianos son ínfimos.

Cabe señalar que en el año 2008, Cuba destinó el 10.9\% del Producto Interno Bruto (PIB) para atender a los beneficiarios de la seguridad social y de la asistencia social. Este es uno de los principales factores que han determinado que la participación de los adultos mayores en la actividad económica refleje tasas de $20 \%$ y $2 \%$ respectivamente, para hombres y mujeres en el año $2000^{11}$. Dichas tasas se encuentran entre las más bajas de América Latina.

Ampliando la información anterior, cabe señalar que ya desde la década los años 80 del siglo pasado, se llevaron a cabo dos revalorizaciones de las pensiones. En 1983 se incrementó en \$4 pesos mensuales a aquellas que tenían una cuantía hasta $\$ 80$ pesos mensuales, otra hasta $\$ 5$ pesos mensuales en aquellas citadas pensiones inferiores a $\$ 100$ pesos. Esta tendencia al incremento de las mismas se mantuvo en la siguiente década. En el año 2005 ocurre otro aumento elevando la mínima hasta $\$ 150$ pesos y en diciembre de 2008 (según la Ley 105) alcanzaron \$200 pesos en tanto que una resolución estableció que a las nuevas que se concedieran se les aplicara una escala que favorecería la cuantía de la misma. La referida ley cambió la edad de jubilación, pasando a ser 65 años para los hombres y de 60 años para las mujeres. Esta se aplicará en un período de transición de 5 años. Un hecho interesante es que las personas que lleven laborando 50 años o más, recibirán el 90\% del promedio de sus mejores ingresos en los últimos años trabajados.

En cuanto a los salarios, éstos también experimentaron cambios recientes, en particular en el 2005 y el 2007. En el primero de ellos se estableció que el salario mínimo

8. Welti, Carlos y Verónica Montes de Oca. 1997: Envejecimiento: Nuevos escenarios para los servicios de salud y seguridad social. En: "PROLAP (Programa Latinoamericano de Población). Población y cambio social. Vol. 1. № 1. México. Maingón, Thais. 1997: "Proceso de privatización en los servicios de salud en América Latina y el Caribe: estudio de casos”. En: PROLAP (Programa Latinoamericano de Población). Población y Cambio Social Vol. I, № 1, México.

9. CELADE-CEPAL (2002): Los adultos mayores en América Latina. Datos e Indicadores. Stgo. de Chile, 2002.

10. Guzmán, J.M. (2002): "Envejecimiento y ... op. cit.

11. United Nations. "Population Ageing 2002". Population Division Department of Economic and Social Affairs. 
de los trabajadores fuera de $\$ 225.00$. En ese propio año por la Resolución 30, se revisaron los calificadores de cargo y se incrementó a cada grupo o escala el sueldo. Con esta medida se favorecieron todos los que laboran en el país. En el otro, se aplicaron beneficios salariales a 4 organismos de la Administración Central del Estado, se les pagó antigüedad, y una mensualidad por trabajar en los mismos (Ministerios de Trabajo y Seguridad Social, de Finanzas y Precios, de Economía y Planificación y MAC) con esto se beneficiaron 3100 personas. Posteriormente, en el año 2008 se le aplicaron incrementos salariales a los que laboraban en los Ministerios de Educación, Educación Superior y además a los Jueces y Fiscales, beneficiándose 65,559 individuos ${ }^{12}$.

En otro orden de cosas, en Cuba, la atención a los hogares de ancianos está incluida en la Ley de Seguridad Social vigente. Ellos tienen acceso a estos hogares a partir de la edad de retiro y sus características básicas son las de tratar de hacerles sentir que están en su casa; además, se pretende que se puedan valer por sí mismos.

Estas unidades de asistencia social del país se han incrementado considerablemente, como parte de los esfuerzos por satisfacer las necesidades de este segmento

Cuadro 9. Cuba: Unidades de Asistencia Social del Minis-
terio de Salud Pública y su dinámica. 1965-2003. Años
seleccionados. Cantidad de unidades y porcentajes.
\begin{tabular}{|l|c|c|c|c|c|c|c|}
\hline Unidades/Años & 1965 & 1970 & 1975 & 1980 & 1984 & 1990 & 2007 \\
\hline $\begin{array}{l}\text { Hogares de } \\
\text { ancianos }\end{array}$ & 29 & 40 & 48 & 74 & 98 & 155 & $370^{*}$ \\
\hline $\begin{array}{l}\text { Hogares de } \\
\text { impedidos físicos } \\
\text { y mentales }\end{array}$ & 1 & 6 & 6 & 10 & 18 & 24 & 37 \\
\hline Dinámica (\%) & 100 & 138 & 166 & 255 & 338 & 534 & 1275 \\
\hline $\begin{array}{l}\text { Hogares de } \\
\text { ancianos }\end{array}$ & 100 & 600 & 600 & 1000 & 1800 & 2400 & 3700 \\
\hline $\begin{array}{l}\text { Hogares de } \\
\text { impedidos físicos } \\
\text { y mentales }\end{array}$ & & & & & & & \\
\hline
\end{tabular}

Fuente: Rodríguez, V. Hernández, R. Puga, D. Características demográficas y socio-económicas del envejecimiento de la población en España y Cuba. Consejo Superior de Investigaciones Científicas. Monografías 26. Madrid, 2003. Pág. 55 y MINSAP, 2008.

(*) Incluye 226 Casas de Abuelos. de la población. Sin embargo, cabe señalar que a pesar del crecimiento que han registrado las referidas unidades, todavía están muy lejos de satisfacer las crecientes necesidades de dicha población, lo cual está asociado, entre otros factores, con el explosivo crecimiento de los adultos mayores en los últimos años (ver cuadro No.9).

Existen otra serie de actividades que desarrollan los adultos mayores, en todas las provincias del país, tales como:

a) Ejercicios físicos sistemáticos en Círculos y Casas de Abuelos y en los Hogares de Ancianos.

b) Participación en Grupos de Orientación y Recreación.

c) Adultos Mayores afiliados a Casas de Cultura que realizan actividades en diferentes manifestaciones artísticas.

Los Círculos de Abuelos constituyen una organización de ancianos con amplios intereses sociales y de carácter autónomo, nacido en la última década del siglo anterior y que se estimula en la aplicación de sus intereses sociales y en el desarrollo de programas de autoayuda y ayuda mutua, entre otros, que contribuyen a que el anciano participe activamente en la solución de sus problemas.

Las Casas de Abuelos brindan atención a ancianos con un grado de discapacidad tal que no puedan mantenerse solos en su hogar y que socialmente no sea posible solucionar esta situación con otras alternativas. Al mismo tiempo estos ancianos, para su admisión en la Casa de Abuelos deben ser capaces de realizar por sí mismos las actividades básicas de la vida diaria. Durante su estancia en estos centros, se trabaja en la búsqueda de soluciones para la reinserción a tiempo completo del anciano en su hogar o prolongar sus posibilidades sin institucionalizar al mismo.

Los aspectos anteriores constituyen sólo una breve referencia a algunas informaciones relativas al sub programa comunitario, las cuales se brindan en el Cuadro No.10. En primer término, se aprecia cómo el monto de

12. Las informaciones de éste y el párrafo anterior fueron suministradas por el Instituto Nacional de Asistencia y Seguridad Social, del Ministerio de Trabajo y Seguridad Social. 


\begin{tabular}{|c|c|c|}
\hline Indicadores & 1998 & 2005 \\
\hline Casas de Abuelos & 74 & 186 \\
\hline Participantes Casas de Abuelos & 2.404 & 5.562 \\
\hline Círculos de Abuelos & 12.229 & 14.701 \\
\hline Participantes Círculos de Abuelos & 325.451 & 745.083 \\
\hline Ancianos solos en atención domiciliaria & 42.994 & 125.136 \\
\hline Grupos de orientación y recreación & 609 & 1.435 \\
\hline $\begin{array}{l}\text { Equipos multidisciplinarios de atención } \\
\text { gerontológica }\end{array}$ & 424 & 435 \\
\hline \multicolumn{3}{|c|}{$\begin{array}{l}\text { Cátedras Universitarias de la Tercera Edad: 29,502 graduados del } 2000 \\
\text { al } 2005\end{array}$} \\
\hline
\end{tabular}

Fuente: Informaciones suministradas por el Ministerio de Salud Pública.

Casas de Abuelos ha aumentado en 2.5 veces, en tanto el número de participantes en ellas lo ha hecho en 2.3 veces. No menos importante ha sido el incremento, tanto del número de Círculos de Abuelos, como el de sus participantes. Asimismo se destaca cómo se ha triplicado el monto de ancianos solos que ha recibido atención domiciliaria.

En el marco de la capacitación de los adultos mayores existen dos cuestiones importantes: Por una parte, la Educación para la Jubilación, dado que la misma es más necesaria para la inserción del adulto mayor en su nueva condición. No menos importante, es la educación opcional (aulas para los adultos mayores) dirigida a aquellas personas retiradas o a aquellas que desean mejorar su conocimiento sobre el arte, la literatura, etc.

En Cuba se creó hace algunos años la Universidad del Adulto Mayor, la cual pertenece a la Cátedra Universitaria del Adulto Mayor de la Universidad de La Habana, en co-auspicio con el Movimiento de Jubilados y Pensionados de la Central de Trabajadores de Cuba y la Asociación de Pedagogos de Cuba. Se encuentra adscripta a la Facultad de Psicología de dicha universidad.

Uno de sus antecedentes se vincula con la existencia de Universidades de la Tercera edad en el mundo, así como acciones culturales y de salud para adultos mayores en instituciones cubanas. Durante el año 2000 se organizó el I Encuentro Nacional de Adultos Mayores.
Para el diseño curricular de la referida Universidad, se llevaron a cabo investigaciones bibliográficas de proyectos con otras universidades de la tercera edad en el mundo, numerosos intercambios con instituciones cubanas que dirigen acciones a favor de los adultos mayores y estudios diagnósticos acerca de las necesidades de superación con personas mayores.

Entre sus objetivos pueden señalarse los siguientes:

- Contribuir a la creación de una nueva cultura del envejecer, que considere la tercera edad como etapa del desarrollo humano, en la cual se despliegan numerosas potencialidades de aprendizaje y contribución social.

- Lograr la superación cultural y científico-técnica en los adultos mayores, de acuerdo a los avances de la ciencia, la tecnología, el arte, la cultura y el pensamiento humano en la actualidad.

- Promover programas en la solución de problemas del desarrollo cultural global y sostenible.

- Comprender las características del proceso de envejecimiento que propicie armónicas relaciones con los coetáneos, la familia, la comunidad y los vínculos laborales, como lugares de transmisión de la experiencia y de apoyo social.

Inicialmente, los módulos temáticos han sido: a) Desarrollo humano; b) Salud para todos; c) Cultura contemporánea; d) Tiempo libre y recreación; e) Seguridad y servicio social y f) Desarrollo individual o auto desarrollo educativo.

Los cursos de carácter presencial se desarrollan a partir de los antes mencionados módulos temáticos, por lo cual esta Universidad no reproduce ningún plan de estudio referido a las carreras universitarias, sino un plan de estudios particular para la superación cultural y científico-técnica de los adultos mayores, de acuerdo a sus intereses cognoscitivos y de actualización general. Entre los temas de mayor interés se encuentran la computación, y los referidos a las ciencias sociales.

Para cursar esta Universidad, no se requieren requisitos de nivel educacional anterior. Por tanto, estudian adultos mayores provenientes del sector azucarero, por- 
tuario, transporte, educacional, salud, comercio, turismo, metalúrgico, cultural y científico, entre otros.

Los beneficiarios directos con este proyecto son los adultos mayores cubanos, especialmente los jubilados sindicalizados, a los cuales se les han brindado cursos presenciales, es decir, a aquellos jubilados que se encuentran agrupados en el Movimiento de Jubilados y Pensionados.

La Universidad del Adulto Mayor se ha generalizado y expandido a todas las provincias del país, e incluso al Municipio Especial Isla de la Juventud de distintas formas. El monto de graduados durante el período 2000-2005 ratifica la importancia que ha adquirido este movimiento.

\section{Reflexiones finales}

El explosivo crecimiento de los adultos mayores en el mundo, con mayor énfasis en los países subdesarrollados debe considerarse como un aporte que recibirá la humanidad, por la contribución que hacen y harán. Es necesario desterrar de la mente de algunos el criterio de que la citada "explosión" constituirá una alevosa carga, sin tener presente las contribuciones que realizan estas personas, las cuales están en capacidad para ayudar a solucionar los problemas de sus comunidades, así como los propios.
Es importante tener en cuenta, que se hace necesario tomar muchas previsiones ante el gran crecimiento de adultos mayores de 75 años y más, en los cuales aumenta el grado de dependencia y la escasa movilidad. En otras palabras, tratar de lograr que el aumento de la longevidad se traduzca no sólo en vivir más años, sino en mejores condiciones y con salud, lo cual es en verdad una meta difícil de alcanzar.

El mayor reto ante las cuestiones antes planteadas lo tienen la inmensa mayoría de los países subdesarrollados, en los cuales es que verdaderamente tendrá lugar un crecimiento explosivo, pero en condiciones menos ventajosas en que lo han enfrentado los países desarrollados. Es decir, los primeros, en general, no cuentan con los recursos necesarios, ni con la infraestructura requerida para atender todas las necesidades a resolver.

Afortunadamente, los adultos mayores cubanos están en muchas mejores condiciones, pues reciben mejor atención, lo cual se vincula con una gran voluntad política. De todas formas, esto no significa que también el país no enfrente un serio reto, pues en unos pocos años el monto de adultos mayores se duplicará. Lo importante, aparte de tomar las previsiones necesarias, es no enfocar esto como problemas insolubles, sino con optimismo y voluntad de abordar positivamente las cuestiones a resolver. 